\title{
sciforum
}

Conference Proceedings Paper

\section{Long-Term Cloud Cover Variations within the Region of Optical Observatories in the South of Eastern Siberia}

\author{
Elena V. Devyatova ${ }^{1,2, *}$ \\ 1 Institute of Solar-Terrestrial Physics SB RAS, Russia, 664033, Irkutsk p/o box 291; Lermontov st., 126a; \\ uzel@iszf.irk.ru \\ 2 Irkutsk State University, Russia, 664033, Irkutsk, Lermontov st., 126, office 319; dekanat@geogr.isu.ru \\ * Correspondence: elenadevyatova4@gmail.com; Tel.: + 79148762328
}

\begin{abstract}
In the south of Eastern Siberia (Russia), there are optical observatories of the Institute of Solar-Terrestrial Physics SB RAS. The observatories are equipped with instruments to observe the Sun, near and deep space. The first one, Sayan Solar Observatory, is located in the mountains at the height of 2,000 m (51.67 N, 100.99 E). The second, Baikal Astrophysical Observatory, is on the southern bank of Lake Baikal (51.86 N, 104.86 E). These two observatories were built in the 60s 70s of the last century. The purpose of this work is to study the long-term dynamics of cloud cover within the region of the optical observatories. We used data of: Monthly mean Total cloud cover (TCC), High cloud cover (HCC), Low cloud cover (LCC) and Medium cloud cover (MCC) values derived from ECMWF ERA-Interim 1979-2018 [1] and Monthly mean TCC values derived from NCEP/NCAR Reanalysis Gaussian Grid 1948-2019 [2]. Both reanalysis projects show a long-term decline in the annual TCC values. For the summer season, negative trend is most obvious and typical for HCC, MCC, and LCC. In our opinion, the causes of the current changes should be searched for in the peculiarities of long-term variability of large-scale atmospheric circulation, particularly, in blocking activity.
\end{abstract}

Keywords: cloud cover; atmospheric circulation; long-term variability; atmospheric blocking

\section{Introduction}

In the south of Eastern Siberia (Russia), there are three optical observatories of the Institute of Solar-Terrestrial Physics of the Siberian Branch of the Russian Academy of Sciences (ISTP SB RAS), Irkutsk. The observatories are equipped with instruments to observe the Sun, near and deep space. The first one, Sayan Solar Observatory, is located in the mountains at the height of 2,000 m near the settlement of Mondy, Buryatia (51.67 N, 100.99 E). The second, Baikal Astrophysical Observatory, is on the southern bank of Lake Baikal near the settlement of Listvyanka (51.86 N, 104.86 E). These two observatories were built in the $60 \mathrm{~s}-70 \mathrm{~s}$ of the last century. The third observatory is now being built in settl. Tory, Buryatia ( $51.78 \mathrm{~N}, 103.00 \mathrm{E})$. Figure 1 shows location of the observatories.

The state of the atmosphere, through which the light passes, is of great importance for optical observations. Important characteristics influencing the efficiency of optical instruments are clear sky frequency, atmospheric transparency, and turbulence. At the time, the south of Eastern Siberia was chosen to install the instruments as the region with a high frequency of clear sky [3]. Conclusions in [3] refer to the USSR territory and are based on information from data sheets of the Hydrometeorological Center of USSR. 
We are interested in studying the long-term dynamics of meteorological characteristics that affect efficiency of telescopes in this region. One of the most common characteristics that are directly relevant to the concept of clear sky frequency is cloud cover. In this work, based on data from ECMWF ERA-Interim and NCEP/NCAR Reanalysis 1, we investigate into the dynamics of cloud cover in the region of observatories over the decades since the first observations began.

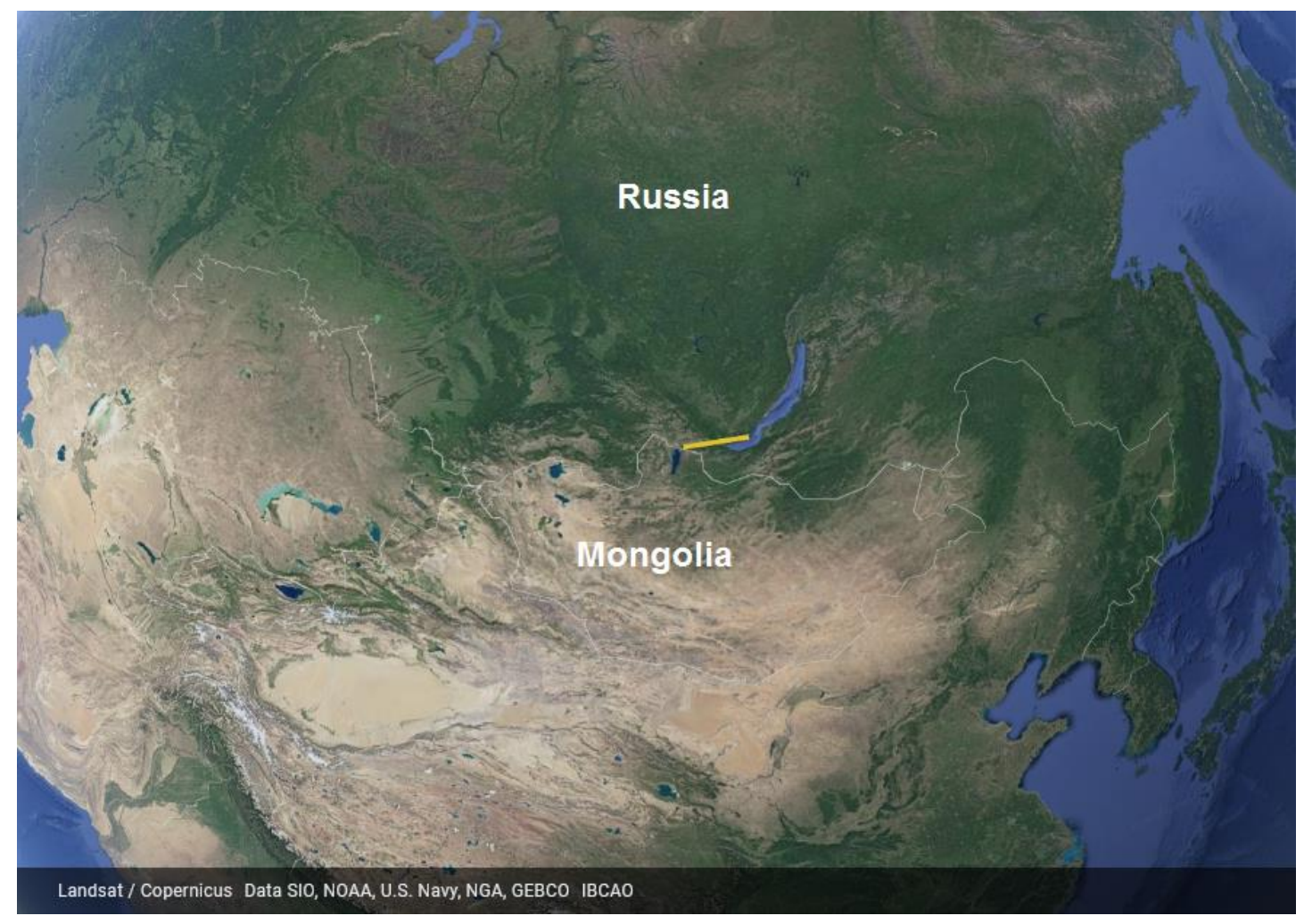

Figure 1. Yellow marker shows the area where optical observatories of ISTP SB RAS are located. https://www.google.com/earth/.

\section{Data and Methods}

We used the following data:

- Monthly mean Total cloud cover (TCC), High cloud cover (HCC), Medium cloud cover (MCC) and Low cloud cover (LCC) values (in \%) derived from ECMWF ERA-Interim 1979-2018 [1] with spatial resolution $1^{\circ} \times 1^{\circ}$.

- Monthly mean TCC values (in \%) derived from NCEP/NCAR Reanalysis 1 Gaussian Grid 1948-2019 [2].

We calculated cloud cover values averaged along 50,475 $\mathrm{N}$ (NCEP) and along $50^{\circ} \mathrm{N}$ (ERA-Interim) over $101^{\circ}-105^{\circ}$ E (5 grid points for ERA and 3 grid points for NCEP). Then, we calculated values of the annual mean cloud cover, seasonal mean cloud cover, and anomalies of the annual mean cloud cover values.

\section{Results}

Figure 2a shows annual mean TCC values in the region of optical observatories of ISTP SB RAS. TCC values from ERA-Interim data are higher than TCC values from NCEP/NCAR Reanalysis. Maximum TCC values over the entire period available for analysis reach 62\% (ERA-Interim) and $51 \%$ (NCEP/NCAR). Minimums are $47 \%$ and $35 \%$ respectively. Both datasets show a gradual decrease in TCC values until about 2010. After 2010, the negative trend in TCC values remains in NCEP/NCAR data, not in ERA-Interim data. 
The 3rd International Electronic Conference on Atmospheric Sciences (ECAS 2020), 16-30 November 2020; Sciforum Electronic Conference Series, Vol. 3, 2020

Figure $2 \mathrm{~b}$ shows the annual mean TCC anomalies calculated relative to the TCC value averaged over 1981-2010. In the 60s-80s of the 20th century, when the observatories were constructed and first observations began, TCC anomalies that we calculated were positive. Since 1990, negative TCC anomalies are prevailing.

Thus, according to ECMWF ERA-Interim and NCEP/NCAR Reanalysis, during first observations at ISTP SB RAS observatories, values of the annual mean TCC were higher than currently. From this point of view, the overall conditions at observatories at present are more favorable than during first observations.

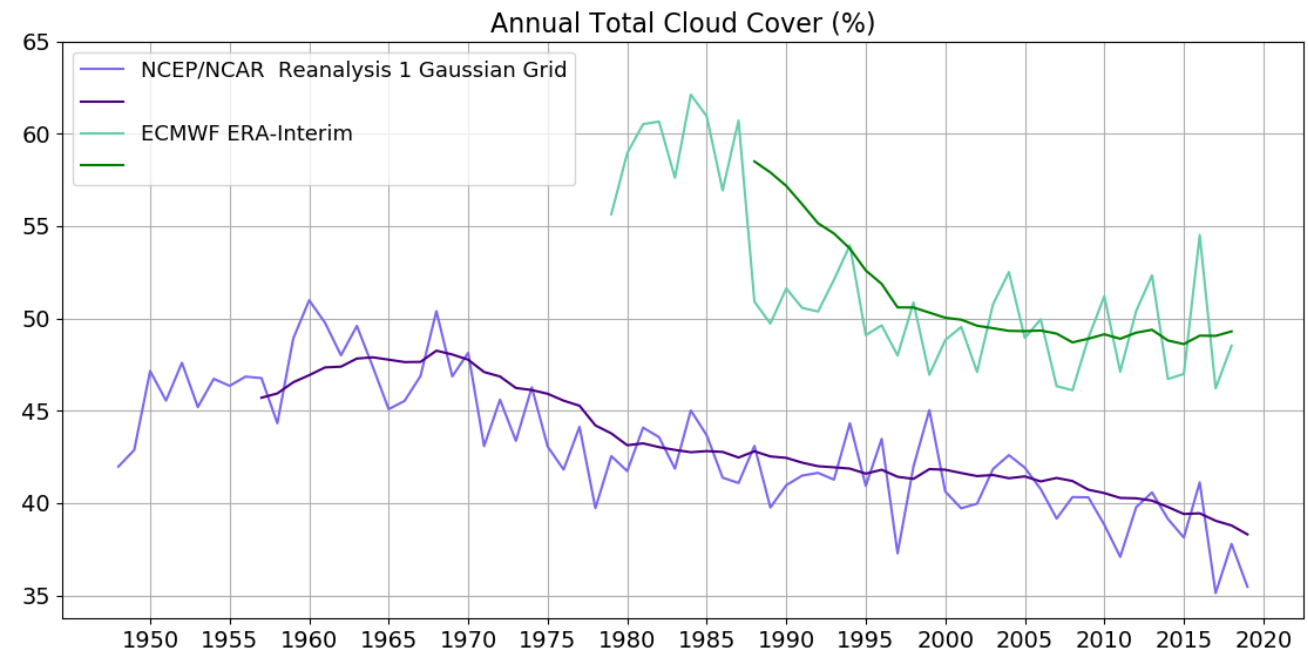

(a)

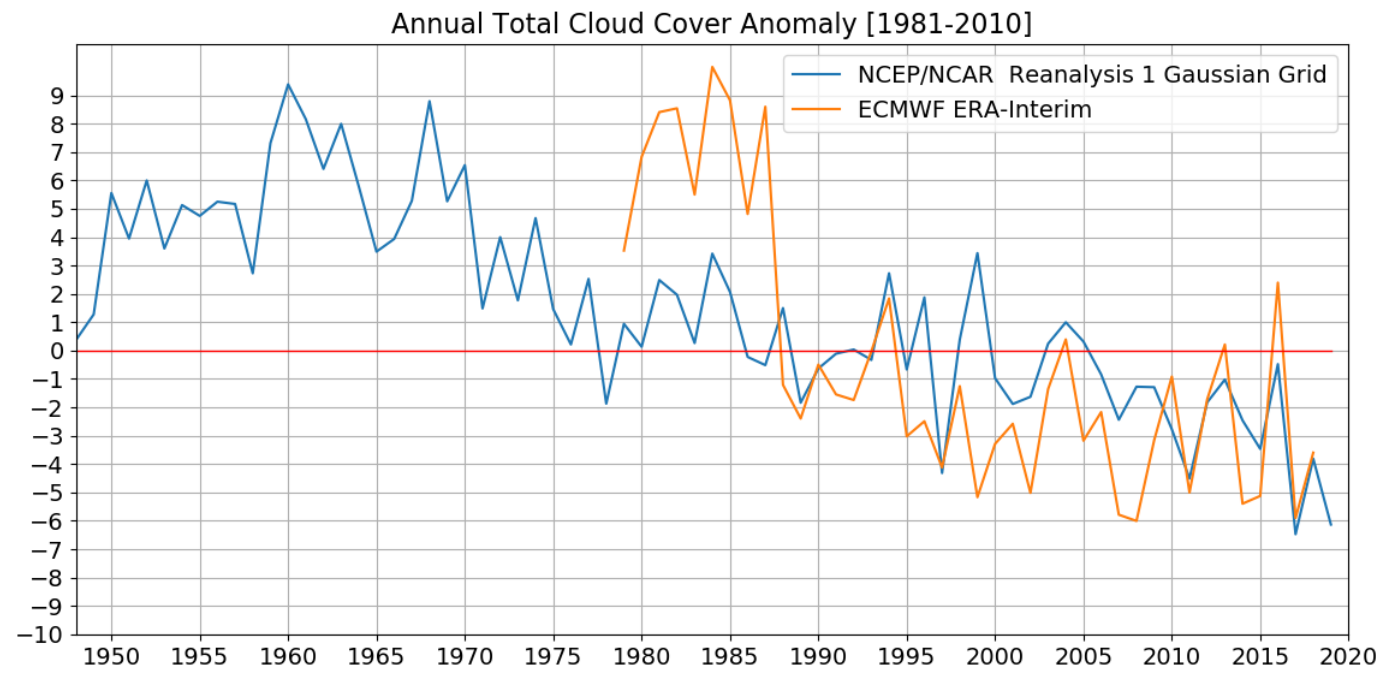

(b)

Figure 2. (a) Annual mean TCC; (b) Annual mean TCC anomalies.

Based on generalities, the second half of spring, summer and early fall seasons in Eastern Siberia are most effective for solar studies in terms of scope and quality of observations. In stellar studies, the largest scope of observations conversely falls on the second half of fall, winter and the first half of spring. Therefore, the next question we would like to ask is in which seasons is the negative trend in TCC values most pronounced? To answer this question, we have plotted the long-term variability of TCC values, averaged over seasons: winter (December, January and February), spring (March, April, May), summer (June, July, August) and fall (September, October, November). 
The 3rd International Electronic Conference on Atmospheric Sciences (ECAS 2020), 16-30 November 2020; Sciforum Electronic Conference Series, Vol. 3, 2020

Figure 3 shows the year-to-year TCC dynamics within the base region of observatories of ISTP SB RAS specifically by seasons according to NCEP/NCAR Reanalysis 1 . The trend towards a long-term decrease in TCC values proved to be most pronounced in summertime. Though there are signs of decreasing TCC values in other seasons of the year. The negative trend of TCC values in all seasons in the last decade is particularly well seen. In our view, this feature can be explained by the phase of the quasi-thirty-year oscillation, which is quite clearly evident in the TCC dynamics in Figure 3. Figure 4 demonstrates the same as Figure 3, only from ECMWF ERA-Interim. According to these data, the trend towards long-term decrease of TCC values is well seen in spring and summer.
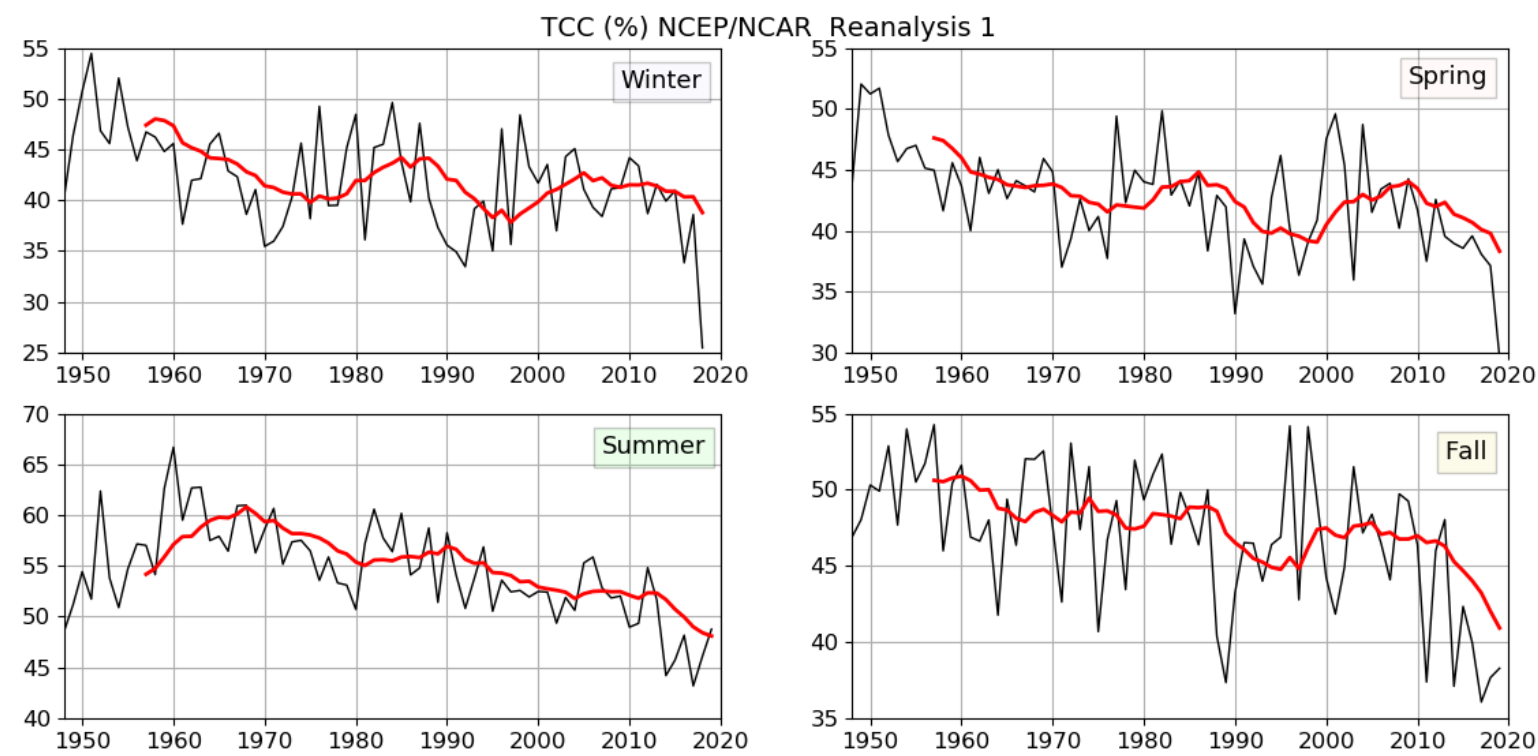

Figure 3. TCC values, averaged over seasons: winter (December, January, February), spring (March, April, May), summer (June, July, August), fall (September, October, November). NCEP/NCAR Reanalysis 1. 1948-2019.
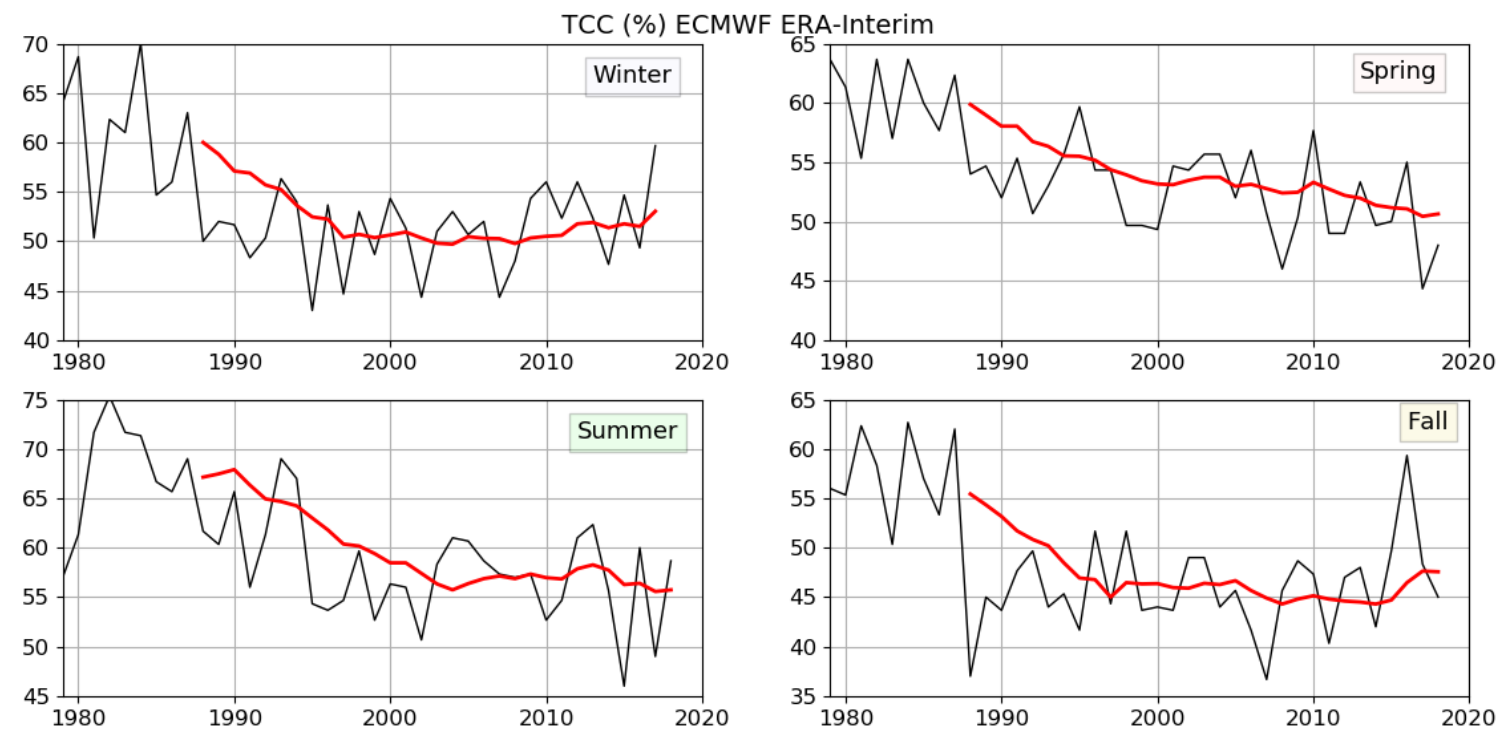

Figure 4. TCC values, averaged over seasons: winter (December, January, February), spring (March, April, May), summer (June, July, August), fall (September, October, November). ECMWF ERA-Interim. 1979-2018. 
The 3rd International Electronic Conference on Atmospheric Sciences (ECAS 2020), 16-30 November 2020; Sciforum Electronic Conference Series, Vol. 3, 2020

In Figure 5, we present plots of long-term cloud cover variations specifically by seasons for High cloud cover (HCC), Medium cloud cover (MCC), and Low cloud cover (LCC) values according to ECMWF ERA-Interim data. Estimation of contribution of different levels to overall changes in cloud cover is important, too. For example, in the practice of solar observations, the author of this paper and colleagues noted that very thin high clouds (Cirrus, Cirrostratus), not only leave the image quality intact, but on the contrary, contribute to its stabilization and better clarity. Variations in the middle and lower clouds is not a matter of quality of observations, but of the very possibility or impossibility of carrying them out. According to Figure 5, in the long-term dynamics, HCC, MCC and LCC behave in a fairly consistent manner. All HCC, MCC, and LCC contribute to the negative TCC trend during the warm period.

Thus, according to ECMWF ERA-Interim and NCEP/NCAR Reanalysis 1, there is a long-term decrease in cloud cover in the region of the ISTP SB RAS observatories during the warm period of the year. Contributing to this trend are the clouds of all three HCC, MCC, and LCC. Obviously, the causes of the current changes should be searched for in the peculiarities of long-term variability of atmospheric circulation in this region.
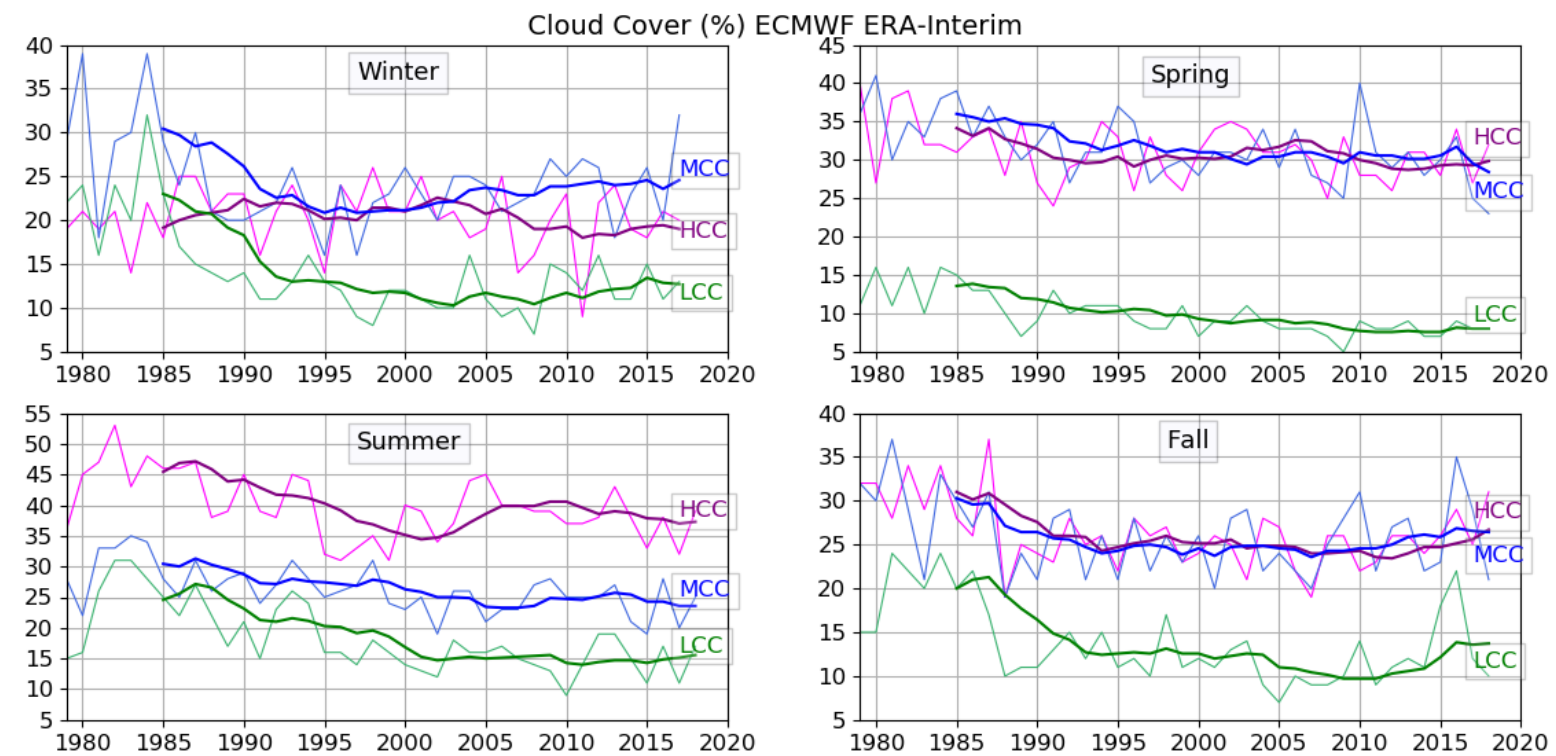

Figure 5. Long-term variations in HCC, MCC and LCC values in different seasons. Data of ECMWF ERA-Interim.

\section{Discussion}

The long-lasting low-water period in the basins of Lake Baikal and its main tributary, the Selenga River is a confirmed fact. Beginning of this period refers to the mid-1990s of the 20th century $[4,5]$. It was found out that the low water in Baikal is due to long-term decrease in the amount of precipitation falling out in summer in the basin of the Selenga River [6-9]. As one of the main reasons for variations in rainfall in the Selenga basin, authors of [7-9] consider the activity of summer blockings in Eastern Siberia, in particular, and in the Eastern hemisphere in general.

The optical observatories of ISTP SB RAS are located close to the basins of Selenga (north and west of it) and Baikal. It is obvious that the influence of large-scale features of atmospheric dynamics will be similar in the Selenga basin and adjacent territories. TCC and precipitation, though not directly, but are closely related. In our paper [10] we compared the year-to-year TCC dynamics derived from ECMWF ERA-Interim and observational data on atmospheric precipitation derivation from GPCC in 1979-2017 for July for the Selenga basin. The results of comparison showed good agreement of long-term dynamics of observational precipitation (GPCC) and model TCC (ERA-interim). Thus, we have a confirmation that TCC from reanalysis projects fairly adequately 
The 3rd International Electronic Conference on Atmospheric Sciences (ECAS 2020), 16-30 November 2020; Sciforum Electronic Conference Series, Vol. 3, 2020

reproduces, at least during the summer period, the long-term downward trend of cloud cover in the region of the observatories revealed in this study.

\section{Conclusions}

From reanalysis projects ECMWF ERA-Interim and NCEP/NCAR Reanalysis 1, we revealed an interesting long-term feature in the dynamics of cloud cover in the region of ISTP SB RAS optical observatories in the south of Eastern Siberia. This feature is the long-term decrease in cloud cover values basically during the summer season. We see several ways for further development of studies of long-term variability of meteorological parameters that influence efficiency of telescopes in the south of Eastern Siberia:

1) Involving other data sets in addition to reanalysis projects.

2) Research into long-term dynamics of other characteristics that on an equal footing with cloud cover determine efficiency of optical instruments - transmissivity of the atmosphere, and turbulence.

3) Study of large-scale features of atmospheric dynamics responsible for variability of meteorological characteristics in the region of our interest. In particular the study of blocking activity.

Funding: The work was performed with budgetary funding of Basic Research program II.16.

Conflicts of Interest: The authors declare no conflict of interest. The funders had no role in the design of the study; in the collection, analyses, or interpretation of data; in the writing of the manuscript, or in the decision to publish the results.

\section{References}

1. Dee, D.P.; Uppala, S. M.; Simmons, A. J. et. al. The ERA-Interim reanalysis: configuration and performance of the data assimilation system. Q. J. R. Met. Soc. 2011, 137, 553-597. https://doi.org/10.1002/qj.828

2. Kalnay, E.; Kanamitsu, M.; Kistler R.et.al. The NCEP/NCAR 40-year reanalysis project. Bull. Amer. Meteor. Soc. 1996, 77, 437-471. https://doi.org/10.1175/1520-0477(1996)077<0437:TNYRP>2.0.CO;2

3. Darchia Sh.P. On the Astronomical Climate of the USSR, Nauka: Moscow, USSR, 1985; pp. 79-113 (in Russian).

4. Frolova, N.L.; Belyakova, P.A.; Grigoriev, V.Y. et.al. Runoff fluctuations in the Selenga River Basin. Regional Environmental Change 2017, 17, 1965-76. https://doi.org/10.1007/s10113-017-1199-0

5. Abasov, N.V.; Bolgov, M.V.; Nikitin, V.M.; Osipchuk, E.N. Level regime regulation in Lake Baikal. Water Resources 2017, 44, 537-546. https://doi.org/10.1134/S1875372812030079

6. Berezhnykh, T.V., Marchenko, O.Y., Abasov, N.V. et al. Changes in the summertime atmospheric circulation over East Asia and formation of long-lasting low-water periods within the Selenga river basin. Geogr. Nat. Resour. 33, 223-229 (2012). https://doi.org/10.1134/S1875372812030079

7. Antokhina, O.Yu.; Antokhin, P.N., et al. Dynamic processes in the atmosphere causing precipitation anomalies in Eastern Siberia and Mongolia in summer period // Fundamental and applied climatology, 2018, N 1, p. 10-27 (in Russian). DOI: 10.21513/2410-8758-2018-1-10-27

8. Antokhina, O.Y. Atmospheric Precipitation within the Selenga River Basin and Large-Scale Atmospheric Circulation Over Eurasia in July. Geogr. Nat. Resour. 2019, 40, 373-383. doi.org/10.1134/S1875372819040097

9. Antokhina, O.Y.; Latysheva, I.V.; Mordvinov, V.I. A Cases Study of Mongolian Cyclogenesis During The July 2018 Blocking Events. Geography, Environment, Sustainability 2019, 12, 66-78. DOI-10.24057/2071-9388-2019-14

10. Devyatova E.V.; Antokhina O.Yu. et.al. Comparison of total cloud cover (ERA-Interim) and precipitation (GPCC) over Mongolia and southern part of Eastern Siberia in July. Proc. SPIE 2019, 11208, doi:10.1117/12.2538640

(C) 2020 by the authors. Submitted for possible open access publication under the terms and conditions of the Creative Commons Attribution (CC BY) license (http://creativecommons.org/licenses/by/4.0/). 
The 3rd International Electronic Conference on Atmospheric Sciences (ECAS 2020), 16-30 November 2020; Sciforum Electronic Conference Series, Vol. 3, 2020 